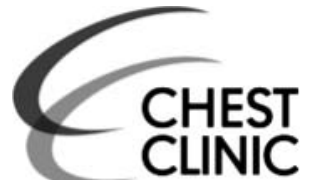

${ }^{1}$ Department of Cardiology, Royal Hallamshire Hospital, Sheffield, UK

${ }^{2}$ Medical Physics Group, Department of Cardiovascular Science, University of Sheffield, Medical School, Sheffield, UK

\section{Correspondence to}

Dr David R Warriner, Medical Physics Group, Department of Cardiovascular Science, I Floor University of Sheffield, Sheffield S10 2JF, UK; d.r.warriner@sheffield.ac.uk

Received 13 July 2012

Accepted 20 September 2012 Published Online First 31 October 2012

\title{
Focal pulmonary oedema: an unusual presentation of acute mitral regurgitation
}

\author{
Paul D Morris, ${ }^{1,2}$ David R Warriner, ${ }^{1,2}$ Kevin S Channer ${ }^{1}$
}

A 46-year-old man presented with dyspnoea, haemoptysis, orthopnoea and a pan-systolic murmur. His chest radiograph (see figure 1) demonstrated asymmetric pulmonary infiltrates, predominantly affecting the right-upper lobe. He was diagnosed with lobar-pneumonia but failed to improve with antibiotic therapy. Echocardiography subsequently demonstrated severe mitral regurgitation (MR), directed towards the right-upper pulmonary vein, secondary to chordal rupture and flail posterior mitral valve leaflet.

Acute MR secondary to a flail posterior mitral valve leaflet often results in a regurgitant jet directed toward the right (usually upper) pulmonary veins. This results in regionalised increased hydrostatic pressure in these veins. This mechanism is supported by selective pulmonary artery wedge pressure measurements and transoesophageal echocardiographic pulmonary vein Doppler data.
Successful surgical repair of the posterior leaflet (thickened, 'Barlow's' valve) resulted in a dramatic improvement. Unilateral pulmonary oedema (typically right-sided) affects up to $9 \%$ of acute MR presentations. Physicians should be aware of this since cases are commonly mistaken for pneumonia.

Other causes of unilateral pulmonary infiltration include pulmonary infarction, pneumonia, malignancy, alveolar haemorrhage, aspiration pneumonitis, foreign body, atelectasis and pulmonary vein obstruction.

Contributors PDM and KSC conceived the idea for the manuscript. PDM and DRW wrote the manuscript. KSC critically revised the manuscript. All authors gave final approval of the version to be published.

Competing interests None.

Patient consent Obtained.

Provenance and peer review Not commissioned; internally peer reviewed.

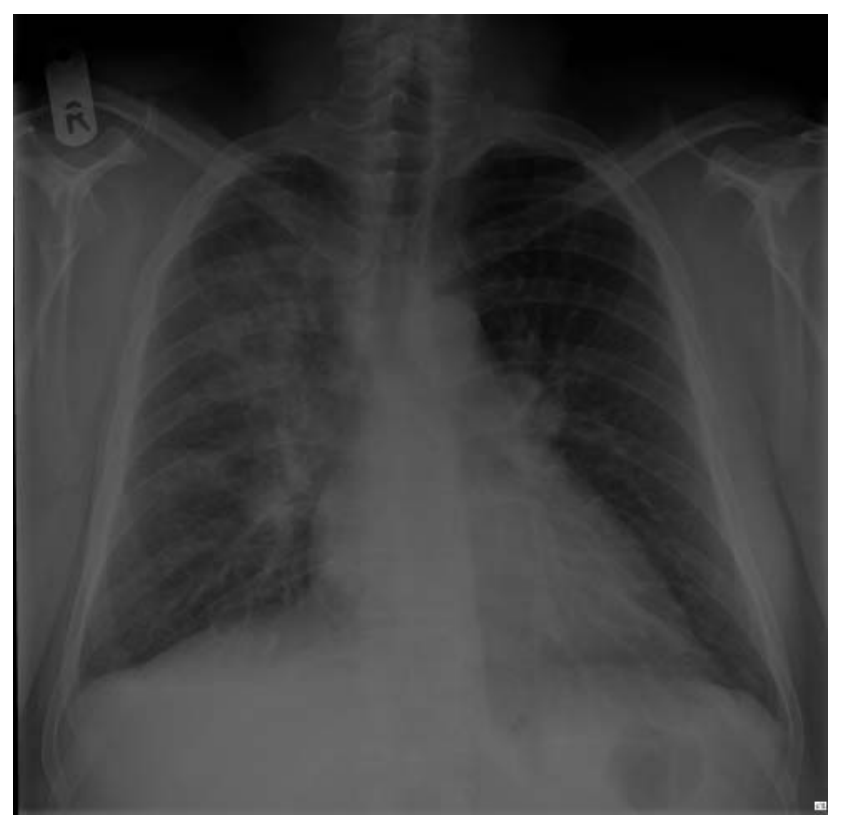

Figure 1 Chest radiograph demonstrating alveolar infiltration of the right lung, predominantly of the upper lobe. 